Article

\title{
Moderately hypofractionated intensity modulated radiotherapy (IMRT) with a simultaneous integrated boost (SIB) for locally advanced head and cancer - Do modern techniques held their promise?
}

\author{
Jörn Wichmann ${ }^{1 *}$, Martin Durisin ${ }^{2}$, Robert Michael Hermann ${ }^{1,3}$, Roland Merten ${ }^{1}$, Hans Christiansen ${ }^{1}$ \\ 1 Hannover Medical School, Department of Radiotherapy, Carl-Neuberg-Str. 1, 30625 Hannover, Germany \\ 2 Hannover Medical School, Department of Otorhinolaryngology, Carl-Neuberg-Str. 1, 30625 Hannover, \\ Germany \\ 3 Center for Radiotherapy, Mozartstr. 30, 26655 Westerstede, Germany \\ * Correspondence: wichmann.joern@mh-hannover.de; Tel.: +49 17615322997
}

Citation: Wichmann, J.; Durisin, M.; Hermann, RM.; Merten, R.; Christiansen H. Moderately hypofractionated intensity modulated radiotherapy (IMRT) with a simultaneous integrated boost (SIB) for locally advanced head and cancer - Do modern techniques held their promise? Curr. Oncol. 2021, 28, FirstpageLastpage.

https://doi.org/10.3390/xxxxx

\begin{abstract}
Background: Intensity modulated radiotherapy (IMRT) is still a standard of care for radiotherapy in locally advanced head and neck cancer (LA-HNSCC). Simultaneous integrated boost (SIB) and moderate hypofractionation offer an opportunity for individual dose painting and a reduction in overall treatment time. We present retrospective data on toxicity and locoregional control of a patient cohort with LA-HNSCC treated with an IMRT-SIB concept in comparison to normofractionated 3D-conformal radiotherapy (3D-RT) after a long-term follow-up.

Methods: Between 2012 and 2014, n=67 patients with HNSCC (stages III/IV without distant metastases) were treated with IMRT-SIB either definitively (single/total doses: 2.2/66 Gy, 2.08/62.4 Gy, 1.8/54 Gy in 30 fractions) or in the postoperative setting (2.08/62.4 Gy, 1.92/57.6 Gy, 1.8/54 Gy). These patients' clinical courses were matched (for sex, primary, and treatment concept) as part of a matched-pair analysis with patients treated before mid-2012 with normofractionated 3D-CRT (definitive: 2/50 Gy followed by a sequential boost up to $70 \mathrm{~Gy}$; postoperative: 2/60-64 Gy). Chemotherapy/ immunotherapy was given concomitantly in both groups in the definitive situation (postoperative dependent on risk factors). The primary endpoints were acute and late toxicity; the secondary endpoint was locoregional control (LRC).
\end{abstract}

Results: Sixty-seven patients treated with IMRT-SIB ( $n=20$ definitive, $n=47$ adjuvant) were matched with 67 patients treated with 3D-RT. There were minor imbalances between the groups concerning nonmatching variables such as extracapsular extension (ECE) and chemotherapy in IMRT-SIB. Significantly less toxicity was found in favor of IMRT-SIB concerning dysphagia, radiation dermatitis, xerostomia, fibrosis, and lymphedema. After a median follow-up of 63 months, the median LRC was not reached (IMRT-SIB) vs. 69.5 months (3D-RT) ( $p=0.63)$.

Conclusions: This moderately hypofractionated IMRT-SIB concept was shown to be feasible with less toxicity than conventional 3D-RT in this long-term follow-up observation.

Keywords: head and neck cancer; radiotherapy; IMRT; SIB; hypofractionation; toxicity 


\section{Introduction}

In locally advanced head and neck cancer (LA-HNSCC), radiotherapy (RT) is an essential element in curative treatment strategies, either in the definitive (in case of inoperability or to avoid mutilating surgery in oropharyngeal cancers) or in the postoperative adjuvant situation [1]. Concomitant - usually cisplatin-based - chemotherapy improves the prognosis in the definitive [2], as well as in the postoperative adjuvant situation, in particular in case of extracapsular spread of nodes (ECE) and/or microscopically involved resection margins [3].

Moderately hypofractionated RT has become more common in clinical practice. It is already the standard of care in postoperative adjuvant RT of breast cancer after breast-conserving surgery [4] and is a guideline-based alternative in RT for prostate cancer [5,6]. Similarly, it has also already been used in HNSCC [7].

Currently, intensity modulated radiotherapy (IMRT) is used as the standard of care in RT for HNSCC to lower the risk of high-grade chronic toxicity [8]. The implementation of IMRT also offers the possibility of simultaneous integrated boost (SIB) radiation for individual dose painting and - using moderately hypofractionated concepts - for reduction of overall treatment time [9].

Here, we present a cohort of patients with locally advanced HNSCC in the curative setting treated with RT or radiochemotherapy (RCT), either definitive or postoperative adjuvant, using an at our institution implemented moderately hypofractionated IMRT-SIB concept. Data on toxicity and locoregional control are reported and compared to a historical cohort of patients treated with normofractionated 3D conformal radiotherapy (3D-RT) before the IMRT era using a matched-pair analysis.

\section{Materials and Methods}

From 2012 to 2014, the 67 analyzed patients with locally advanced (stage III/IV without distant metastases; mostly squamous cell carcinomas) cancers were treated at our department with RT in curative intent with the following moderately hypofractionated IMRT-SIB concepts. For definitive RCT, we used a slightly modified fractionation as proposed by RTOG 0022: 66 Gy (daily dose 2.2 Gy) for gross tumor volumes, 62.4 Gy (2.08 Gy) for elective cervical nodes considered to be at exceptionally high risk for subclinical disease, and $54 \mathrm{~Gy}(1.8 \mathrm{~Gy})$ for elective cervical nodes. In case of postoperative adjuvant treatment, $62.4 \mathrm{~Gy}$ (daily dose $2.08 \mathrm{~Gy}$ ) for the primary tumor region and cervical regions with involved nodes with ECE, 57.6 Gy (1.92 Gy) for cervical regions with involved nodes without ECE, and $54 \mathrm{~Gy}(1.8 \mathrm{~Gy})$ for elective cervical nodes were given. All patients were immobilized with a thermoplastic mask, including the head, neck and shoulder regions. A planning CT scan with a minimum slice thickness of $3 \mathrm{~mm}$ was obtained in all patients. On each CT slice, the gross tumor volumes (GTVs) were delineated by the treating physician, as well as the areas at especially high risk of potential microscopic disease and other potentially affected regions, including lymph nodes (CTVs). The margins to compensate for setup variability and organ motions were mostly $5 \mathrm{~mm}$. Furthermore, organs at risk (OARs), such as the parotids, spinal cord, brachial plexus, and brainstem, were drawn. All calculations were performed with Monaco® (Electa) by experienced physicists. A phantom measurement with the PTW OCTAVIUS® (4D) Phantom (PTW Freiburg GmbH - Germany) and the corresponding PTW VeriSoft ${ }^{\circledR}$ in the latest available version was performed to verify each plan. Planning objectives such as dose prescriptions and normal tissue constraints had to be realized according to ICRU-83 [10] and to QUANTEC data (spinal cord Dmax < 45 Gy, brachial plexus Dmax < 54 Gy, contralateral parotid gland Dmean < 23 Gy) [11]. To reach these objectives/constraints, the PTV coverage was modified if necessary. For RT, linear accelerators with 6 MV photon energy were used.

For comparison, another cohort was additionally analyzed using a matched-pair analysis. The 67 patients of this cohort were treated before the IMRT era at our institution 
from 2008 to 2012 with normofractionated 3D-RT as follows: primary and involved as well as elective cervical nodes up to a dose of $50 \mathrm{~Gy}$ followed by a sequential boost to the primary and involved nodes (or regions with ECE in case of adjuvant therapy) to a total dose of 70 (definitive) or 60 to $64 \mathrm{~Gy}$ (postoperative adjuvant). Planning was performed by multifield 3D conformal forward planning using 6, 10, and $15 \mathrm{MV}$ photon beams and a "shrinking field approach". Dose prescriptions had to be realized according to ICRU-50. To avoid long-term toxicity, the supraclavicular lymph nodes were mostly spared at 46 to $54 \mathrm{~Gy}$, and the spinal cord was spared at $45 \mathrm{~Gy}$.

In both cohorts, concomitant chemotherapy or immunotherapy was given regularly in the definitive situation and in the postoperative adjuvant setting in cases of ECE or microscopically involved resection margins.

All patients were clinically assessed weekly during RT and three months later by experienced staff to evaluate and grade acute toxicity (oral mucositis, dysphagia, radiation dermatitis) according to CTCAE v4.03 [12]. Afterward, patients were asked to show up for yearly follow-up visits to score late toxicities according to LENT/SOMA (xerostomia, taste alteration, fibrosis, lymphedema, hoarseness, fistula, necrosis of mandible, and trismus) [13] to generate a long-term follow-up observation, since late toxicity partly occurs some years later.

For this retrospective matched-pair analysis, approximately 200 consecutive patients who received and completed 3D-RT with curative intent for HNSCC between 01.2008 and 05.2012 were screened as controls. Furthermore, 67 consecutive patients who received hypofractionated IMRT-SIB between 06.2012 and 04.2014 were documented. To select 3D patients as controls, three variables had to match between both groups: sex, site of the primary tumor, and treatment concept (definitive vs. postoperative). Demographic, histopathological, clinical, and toxicity data were collected retrospectively from the charts. Staging was performed according to the 7th version of the TNM manual.

After data collection, for comparison between the patients' characteristics in both cohorts, the McNemar test for binary characteristics or Cohen's kappa for characteristics with more levels were used. Locoregional control (LRC) was analyzed using the Kaplan-Meier method [14]. Differences in toxicity were tested for statistical significance with the Wilcoxon signed-rank test. We used SPSS Version 26 to perform the statistical analyses. Significance was defined as $\mathrm{p}<0.05$.

\section{Results}

Overall, in each group, 67 patients were analyzed (20 definitive, 47 postoperative in each cohort). Median follow-up for all patients was 63 months. The essential patient characteristics are summarized in Table 1 . They were sufficiently balanced between the groups. However, there were significantly more patients with ECE (27\% vs. $8 \%$ ) and with concomitant chemotherapy (70\% vs. $49 \%$ ) treated in the IMRT-SIB cohort, and six vs. three patients suffered from $\mathrm{cN} 2 \mathrm{c}$ disease. 
Table 1 Patients and Tumor Characteristics

\begin{tabular}{|c|c|c|c|c|}
\hline Variable & Indicator & 3D-RT & IMRT-SIB & $\begin{array}{l}\text { McNemar or } \\
\text { Cohens Kappa** } \\
\text { (p-Value) }\end{array}$ \\
\hline \multirow[t]{2}{*}{ Sex } & male & $53(79 \%)$ & $53(79 \%)$ & $\mathrm{p}=1.000$ \\
\hline & female & $14(21 \%)$ & $14(21 \%)$ & \\
\hline \multirow[t]{6}{*}{ Tumor subsite } & Oral cavity & $24(36 \%)$ & $24(36 \%)$ & 1.000 \\
\hline & Oropharynx & $16(24 \%)$ & $16(24 \%)$ & $(\mathrm{p}<0.005)$ \\
\hline & Hypopharynx & $6(9 \%)$ & $6(9 \%)$ & \\
\hline & Larynx & $8(12 \%)$ & $8(12 \%)$ & \\
\hline & Great glands & $5(8 \%)$ & $5(8 \%)$ & \\
\hline & CUP & $8(12 \%)$ & $8(12 \%)$ & \\
\hline \multirow[t]{2}{*}{ Treatment concept } & Definitive & $20(30 \%)$ & $20(30 \%)$ & $\mathrm{p}=1.000$ \\
\hline & Adjuvant & 47 (70\%) & $47(70 \%)$ & \\
\hline \multirow[t]{2}{*}{ Age } & $<65$ & 47 (70\%) & $48(72 \%)$ & \\
\hline & $>65$ & $20(30 \%)$ & $19(28 \%)$ & \\
\hline \multirow[t]{2}{*}{ Histology } & SCC & $62(93 \%)$ & $59(88 \%)$ & 0.423 \\
\hline & Other & $5(7 \%)$ & $8(12 \%)$ & $(p<0.005)$ \\
\hline \multirow[t]{5}{*}{ Grade } & G1 & $0(0 \%)$ & $2(3 \%)$ & \\
\hline & $\mathrm{G} 2$ & $39(58 \%)$ & $35(52 \%)$ & -0.058 \\
\hline & G3 & $27(40 \%)$ & $26(39 \%)$ & $(p=0,619)^{* * *}$ \\
\hline & G4 & $0(0 \%)$ & $1(2 \%)$ & \\
\hline & GX & $1(2 \%)$ & $3(5 \%)$ & \\
\hline \multirow[t]{3}{*}{ UICC stage } & III & $16(24 \%)$ & $15(22 \%)$ & 0.579 \\
\hline & IVA & $47(70 \%)$ & $46(69 \%)$ & $(\mathrm{p}<0.005)$ \\
\hline & IVB & $4(6 \%)$ & $4(6 \%)$ & \\
\hline \multirow[t]{10}{*}{ T stage } & cT1 & 1 & 0 & \\
\hline & cT2 & 0 & 0 & \\
\hline & cT3 & 4 & 6 & \\
\hline & cT4/4a & 13 & 14 & \\
\hline & cT4b & 2 & 0 & \\
\hline & pT1 & 13 & 7 & \\
\hline & pT2 & 10 & 14 & \\
\hline & pT3 & 5 & 9 & \\
\hline & $\mathrm{pT} 4 / 4 \mathrm{a}$ & 11 & 8 & \\
\hline & pT4b & 0 & 0 & \\
\hline \multirow[t]{7}{*}{ N stage } & cN0 & 5 & 8 & \\
\hline & $\mathrm{cN} 1$ & 1 & 0 & \\
\hline & cN2a & 0 & 1 & \\
\hline & $\mathrm{cN} 2 \mathrm{~b}$ & 5 & 4 & \\
\hline & $\mathrm{cN} 2 \mathrm{c}$ & 3 & 6 & \\
\hline & $\mathrm{cN} 3$ & 1 & 1 & \\
\hline & pN0 & 9 & 11 & \\
\hline
\end{tabular}




\begin{tabular}{lllll} 
& pN1 & 13 & 7 & \\
& pN2a & 5 & 5 & \\
& pN2b & 15 & 16 & \\
& pN2c & 6 & 5 & \\
Resection Status & pN3 & 2 & 3 & 0.614 \\
& R0 & $34(51 \%)$ & $39(58 \%)$ & $(\mathrm{p}<0.005)$ \\
& R1 & $6(9 \%)$ & $4(6 \%)$ & \\
Treatment for re- & R2 & $0(0 \%)$ & $1(2 \%)$ & \\
lapset & Yes & $5(8 \%)$ & $3(5 \%)$ & \\
ECE & No & $12(18 \%)$ & $6(9 \%)$ & \\
& Yes & $55(82 \%)$ & $61(91 \%)$ & \\
& No & $5(8 \%)$ & $18(27 \%)$ & \\
Chemotherapy & Not examined & $15(22 \%)$ & $20.001^{*}$ \\
& Yes & $33(49 \%)$ & $47(70 \%)$ & $\mathrm{p}=0.007^{*}$ \\
& No & $34(51 \%)$ & $20(30 \%)$ & \\
\hline
\end{tabular}

* Statistically significant difference between the groups

** Cohens Kappa $<0.00$ poor agreement, $0.00-0.20$ slight, $0.21-0.40$ fair, $0.41-0.60$ moderate, $0.61-0.80$ substantial, $0.81-1.00$ almost perfect according to (30)

*** Negative Cohens Kappa cannot be interpreted, there for there is no statistical sig nificance

† The current treatment was due to a relaps.

Acute and chronic toxicity are shown in Table 2. There were no significant differences in acute oral mucositis incidences, although there were slightly more patients with at least grade 3 mucositis with 3D-RT ( $48 \%$ vs. $40 \%$ ). However, a statistically significant difference was documented with lower toxicity in the IMRT-SIB group for overall dysphagia ( $\mathrm{p}=0.044)$ and radiation dermatitis $(\mathrm{p}=0.002)$.

Concerning late toxicity, xerostomia, fibrosis and edema were significantly lower in the IMRT-SIB group. Nine percent of IMRT-SIB patients vs. nearly $60 \%$ were suffering from xerostomia grade $2 / 3,7.5 \%$ had fibrosis grade $1 / 2$ vs. approximately $34 \%$, and edema grade $2 / 3$ was documented in $11.9 \%$ vs. $44.8 \%$.

3-year LRC was 77\% (SIB-IMRT) vs. 78\% (3D-RT). The median LRC was not reached (SIB-IMRT) vs. $69.5 \mathrm{~m}(\log$ rank 0.23, $\mathrm{p}=0.63)$. 
Table 2 Acute and late toxicity according to CTCAE and LENT-SOMA classification, worst observed during the follow-up

\begin{tabular}{|c|c|c|c|c|c|}
\hline Acute toxicity & & IMRT-group & Control-group & Statistics* & \\
\hline \multirow[t]{6}{*}{ Mucositis } & Grade 0 & $1(1.5 \%)$ & 0 & Z-value (Wilcoxon) & -0.688 \\
\hline & Grade I & $6(9.0 \%)$ & $7(10.4 \%)$ & $\mathrm{p}$-value & 0.492 \\
\hline & Grade II & $33(49.3 \%)$ & $28(41.8 \%)$ & $\mathrm{n}$ & 67 \\
\hline & Grade III & $27(40.3 \%)$ & $32(47.8 \%)$ & $\mathrm{r}$ & 0.08 \\
\hline & Grade IV & 0 & 0 & comment & non-significant \\
\hline & median & 2 & 2 & & \\
\hline \multirow[t]{6}{*}{ Dermatitis } & Grade 0 & $1(1.5 \%)$ & 0 & Z-value (Wilcoxon) & -3.024 \\
\hline & Grade I & $49(73.1 \%)$ & $40(59.7 \%)$ & $\mathrm{p}$-value & 0.002 \\
\hline & Grade II & $17(25.4 \%)$ & $22(32.8 \%)$ & $\mathrm{n}$ & 67 \\
\hline & Grade III & 0 & $5(7.5 \%)$ & $\mathrm{r}$ & 0.37 \\
\hline & Grade IV & 0 & 0 & comment & significant, \\
\hline & median & 1 & 1 & & medium effect \\
\hline \multirow[t]{6}{*}{ Dysphagia } & Grade 0 & $9(13.4 \%)$ & $1(1.5 \%)$ & Z-value (Wilcoxon) & -2.014 \\
\hline & Grade I & $9(13.4 \%)$ & $6(9.0 \%)$ & $\mathrm{p}$-value & 0.044 \\
\hline & Grade II & $20(29.9 \%)$ & $28(41.8 \%)$ & $\mathrm{n}$ & 66 \\
\hline & Grade III & $28(41.8 \%)$ & $32(47.8 \%)$ & $\mathrm{r}$ & 0.25 \\
\hline & Grade IV & 0 & 0 & comment & significant, \\
\hline & median & 2 & 2 & & medium effect \\
\hline Late toxicity & & IMRT-group & Control-group & Statistics & \\
\hline \multirow[t]{6}{*}{ Xerostomia } & Grade 0 & $3(4.5)$ & $4(6.0 \%)$ & Z-value (Wilcoxon) & -4.029 \\
\hline & Grade I & $43(64.2 \%)$ & $10(14.9 \%)$ & $\mathrm{p}$-value & 0.000 \\
\hline & Grade II & $6(9.0 \%)$ & $32(47.8 \%)$ & $\mathrm{n}$ & 45 \\
\hline & Grade III & 0 & $8(11.9 \%)$ & $\mathrm{r}$ & 0.60 \\
\hline & Grade IV & 0 & 0 & comment & significant, \\
\hline & median & 1 & 2 & & large effect \\
\hline \multirow[t]{6}{*}{ Fibrosis } & Grade 0 & $47(70.1 \%)$ & $31(46.3 \%)$ & Z-value (Wilcoxon) & -3.554 \\
\hline & Grade I & $5(7.5 \%)$ & $18(26.9 \%)$ & $\mathrm{p}$-value & 0.000 \\
\hline & Grade II & 0 & $5(7.5 \%)$ & $\mathrm{n}$ & 45 \\
\hline & Grade III & 0 & 0 & $\mathrm{r}$ & 0.53 \\
\hline & Grade IV & 0 & 0 & comment & significant, \\
\hline & median & 0 & 0 & & large effect \\
\hline \multirow[t]{6}{*}{ Hoarseness } & Grade 0 & $34(50.7 \%)$ & $38(56.7 \%)$ & Z-value (Wilcoxon) & -0.220 \\
\hline & Grade I & $15(22.4 \%)$ & $9(13.4 \%)$ & $\mathrm{p}$-value & 0.826 \\
\hline & Grade II & 0 & $6(9.0 \%)$ & $\mathrm{n}$ & 44 \\
\hline & Grade III & $1(1.5 \%)$ & $1(1.5 \%)$ & $r$ & 0.03 \\
\hline & Grade IV & 0 & 0 & comment & non-significant \\
\hline & median & 0 & 0 & & \\
\hline \multirow[t]{2}{*}{ Taste alteration } & Grade 0 & $20(29.9 \%)$ & $22(32.8 \%)$ & Z-value (Wilcoxon) & -1.136 \\
\hline & Grade I & $28(41.8 \%)$ & $20(29.9 \%)$ & $\mathrm{p}$-value & 0.256 \\
\hline
\end{tabular}




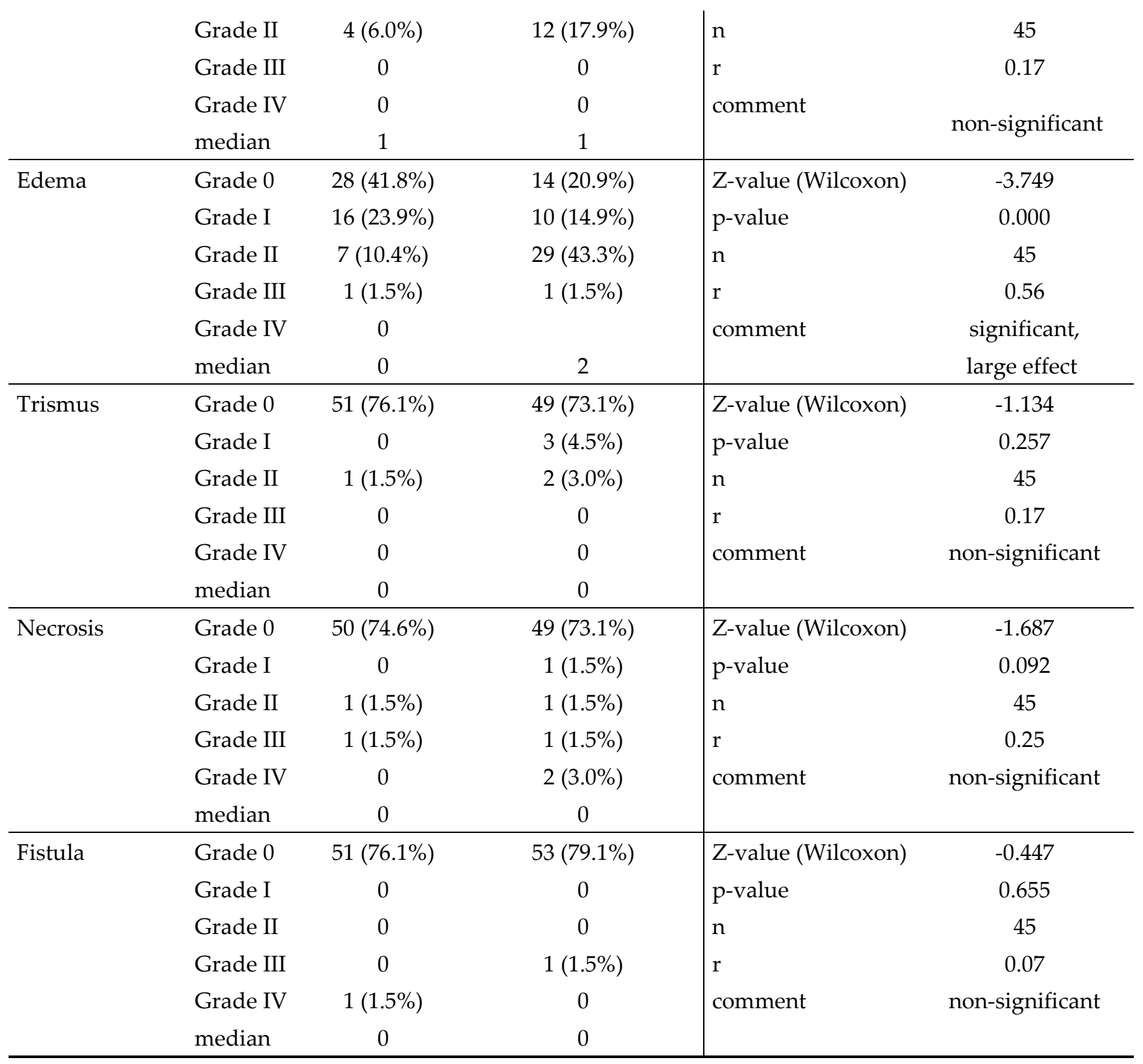

* Reported statistics: Z-value - test statistic of the Wilcoxon signed-rank test; p-value significance $<0.05 ; \mathrm{n}$ - number of pairs, which have both reported adverse events; $\mathrm{r}$ - effect size, calculated by $\boldsymbol{r}=\frac{Z}{\sqrt{N}}$; comment: $\mathrm{r}<0.3$ small effect, $\mathrm{r}$ between 0.3 and 0.5 medium effect, $r>0.5$ large effect according to Cohen

\section{Discussion}

With the advent of IMRT in RT for HNSCC as a standard of care, diverse individual concepts have been implemented in different radiotherapy departments. Here, the application of an SIB and moderate hypofractionation are often applied to ensure individual dose painting and reduction of overall treatment time, which is crucial in RT for HNSCC [15].

To our knowledge, there is little prospective randomized evidence to evaluate the efficacy of IMRT in comparison to 3D-RT in the RT of HNSCC. One study (PASPORT) randomized $n=94$ patients between IMRT and 3D-RT (with parallel opposed lateral fields) [16]. This study was focused on avoiding xerostomia. At 12 and 24 months, xero- 
stomia of at least grade 2 was significantly less prevalent after IMRT. Other late toxicities and locoregional control or overall survival did not differ between the groups. Comparable results were obtained in a small randomized trial ( $n=60$ patients) from India [17].

In nasopharyngeal cancer, this advanced technique has demonstrated a higher oncological efficacy in $n=616$ patients compared to outdated 2D planning techniques: OS and progression-free survival were significantly improved. At the same time, high-grade chronic toxicity was reduced [18].

The recently published GORTEC 2004-01 randomized phase III trial again showed that the IMRT technique can even reach a dose escalation with markedly decreased late xerostomia but without a significant improvement of local tumor control [31]. The authors used a slightly different irradiation concept with a sequential moderate hypofractionated boost to $75 \mathrm{~Gy}$ overall dose ( $25 \mathrm{~Gy} / 10 \mathrm{~F}$ boost dose) in a total of 35 fractions. The frequency of grade $\geq 2$ xerostomia was approximately $2 / 3$ lower after 1 and 3 years in the IMRT group.

Finally, Gupta et al. repeatedly showed in a prospective randomized trial with a very long follow-up and sufficient power and sample size that moderate hypofractionation with 66 Gy in 30 fractions and the IMRT technique led to a meaningful reduction in severe xerostomia and fibrosis with comparable locoregional control and overall survival in the 3D control group [32].

Other observational studies support the assessment of lower acute and chronic toxicity by IMRT in RT for HNSCC in comparison to 3D planning. In this context, Jirkovska et al. demonstrated that acute toxicity and xerostomia were significantly reduced in HNSCC treated by IMRT [19]. Modesto et al. showed similar data, especially for severe late toxicities such as xerostomia, dysphagia, or feeding-tube dependency [20]. Our data confirm these findings, showing lower toxicity in the IMRT-SIB group for dysphagia, dermatitis, xerostomia, fibrosis, and edema.

Other retrospective studies also showed an advantage for IMRT concerning prognosis in LRC [21] or even OS [22]. In contrast, in our patients, LRC was equal between the two groups. Despite the matched pair analysis, other biases due to the study's retrospective nature might play a role. However, such a finding is not totally in conflict with the literature. A recent meta-analysis on IMRT versus 3D-CRT in RT for HNSCC confirmed the superiority of IMRT in terms of toxicity (mainly xerostomia) but did not find improved oncological outcomes. The authors concluded that a positive impact of IMRT on tumor control (and survival) remains to be proven [8].

As concomitant chemotherapy in RT for locally advanced HNSCC is crucial for prognosis in definitive and specific postoperative adjuvant situations, RT approaches must be designed so that the dose and fractionation concepts do not compromise the use of concurrent systemic therapy and vice versa. Therefore, we chose mostly a chemotherapy protocol with weekly low-dose cisplatin $\left(40 \mathrm{mg} / \mathrm{m}^{2}\right.$ BSA up to at least cumulative $\geq 200 \mathrm{mg} /{ }^{2} \mathrm{BSA}$, see above) as a continuous radiosensitizer and decided against a higher hypofractionated RT schedule $>2.2$ Gy in the volume. Such a low-dose weekly cisplatin application is an established regimen [23] in addition to the often used high-dose application, for example, $100 \mathrm{mg} / \mathrm{m}^{2}$ BSA twice or thrice during radiotherapy [3]. A cumulative cisplatin dose of approximately $200 \mathrm{mg} / \mathrm{m}^{2} \mathrm{BSA}$, independent of the schedule, might be sufficient to yield a beneficial antitumor effect [24]. However, prospective studies in adequately sized phase III trials on this subject are still pending [25]. We observed good tolerance and feasibility of our approach with moderate hypofractionation without compromising one part of the combined treatment. Other studies actually show that higher hypofractionation (with single doses in SIB volumes up to 2.25 or $2.4 \mathrm{~Gy}$ ) combined with chemotherapy seems to be possible [26, 27].

IMRT-SIB RT concepts will further be modified according to human papillomavirus (HPV) status in locally advanced HNSCC. On the one hand, HPV-positive tumors have a better prognosis and are possible candidates for dose reduction, which is the subject of several ongoing clinical trials [28]. On the other hand, other studies examine the feasibility of dose-escalated hypofractionated chemoradiation in HPV-negative cancer [29]. 
Unfortunately, in our retrospective patient cohorts, HPV status was not available for most of the tumors.

In summary, the presented moderately hypofractionated IMRT-SIB concept was feasible with acceptable locoregional control and less toxicity than conventional 3D-CRT. IMRT is the standard of care in RT for locally advanced HNSCC. The optimal dose/fractionation concept concerning moderate hypofractionation still has to be defined.

Author Contributions: Conceptualization, J.W. and C.H.; methodology, J.W. and C.H.; software, J.W.; formal analysis, J.W.; writing - original draft preparation, J.W., RM. H., C.H.; writing - review and editing, M.D. and R.M. All authors have read and agreed to the published version of the manuscript.

Funding: This research received no external funding.

Institutional Review Board Statement: The study was conducted according to the guidelines of the Declaration of Helsinki, and approved by the Institutional Ethics Committee of Hannover Medical School (protocol code 1795-2013).

Informed Consent Statement: Informed consent was obtained from all subjects involved in the study.

Data Availability Statement: Data is contained within the article.

Acknowledgments: We would like to thank Loukia Spineli for her assistance with the analysis plan at the earlier stages of the study.

Conflicts of Interest: The authors declare no conflict of interest. 
1. Marur S., Forastiere A.A. Head and Neck Squamous Cell Carcinoma: Update on Epidemiology, Diagnosis, and Treatment. Mayo Clin Proc 2016, 91:386-396.

2. $\quad$ Pignon J.P., le Maître A., Maillard E. et al. Meta-analysis of chemotherapy in head and neck cancer (MACH-NC): an update on 93 randomized trials and 17.346 patients. Radiother Oncol 2009, 92:4-14.

3. Bernier J., Cooper J.S., Pajak T.F. et al. Defining risk levels in locally advanced head and neck cancers: a comparative analysis of concurrent postoperative radiation plus chemotherapy trials of the EORTC (\#22931) and RTOG (\# 9501). Head Neck 2005, 27:843-850.

4. Interdisziplinäre S3-Leitlinie für die Früherkennung, Diagnostik, Therapie und Nachsorge des Mammakarzinoms. $\begin{array}{lllllll}\text { Langversion } & 4.3 & - & \text { Februar } & 2020 & \text { AWMF-Registernummer: }\end{array}$ https://www.leitlinienprogramm-onkologie.de/index.php?id=67\&type=0. Accessed 30 August 2020

5. Interdisziplinäre Leitlinie der Qualität S3 zur Früherkennung, Diagnose und Therapie der verschiedenen Stadien des

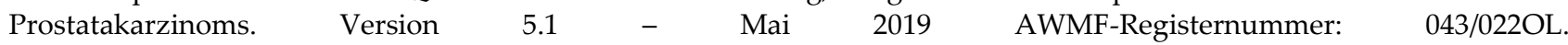
https://www.leitlinienprogramm-onkologie.de/index.php?id=58\&type=0. Accessed 30 August 2020

6. Vassis S, Nöldeke B, Christiansen H et al (2020) Moderately HRT vs. CRT for localized prostate cancer using image-guided VMAT with SIB: evaluation of acute and late toxicities. Strahlenther Onkol 196:598-607

7. Franzese C., Fogliata A., Franceschini D. et al. (2020) Impact of hypofractionated schemes in radiotherapy for locally advanced head and neck cancer patients. Laryngoscope 2020, 130:E163-170

8. Felice F., Pranno N., Papi P. et al. Xerostomia and Clinical Outcomes in Definitive Intensity Modulated Radiotherapy (IMRT) Versus Three-dimensional Conformal Radiotherapy (3D-CRT) for Head and Neck Squamous Cell Carcinoma: A Meta-analysis. Vivo 2020, 34:623-629.

9. Orlandi E., Palazzi M., Pignoli E. et al. Radiobiological basis and clinical results of the simultaneous integrated boost (SIB) in intensity modulated radiotherapy (IMRT) for head and neck cancer: A review. Crit Rev Oncol Hematol 2010, 73:111-125.

10. ICRU Prescribing, Recording, and Reporting Photon-Beam Intensity-Modulated Radiation Therapy (IMRT). Journal of the ICRU 2010, 10: Report 83.

11. Bentzen S.M., Constine L.S., Deasy J.O. et al. Quantitative Analyses of Normal Tissue Effects in the Clinic (QUANTEC): an introduction to the scientific issues. Int J Radiat Oncol Biol Phys 2010, 76(3 Suppl):S3-S9.

12. NCI Common Terminology Criteria for Adverse Events (CTCAE). https://evs.nci.nih.gov/ftp1/CTCAE/About.html. Accessed 1st March 2021.

13. LENT SOMA tables. Radiother Oncol 1995, 35:17-60.

14. Kaplan E., Meier P. Nonparametric estimation from incomplete observations. J Am Stat Assoc 1958, 58:457-481.

15. Dahlke S., Steinmann D., Christiansen H. et al. Impact of Time Factors on Outcome in Patients with Head and Neck Cancer Treated with Definitive Radio(Chemo)Therapy. In Vivo 2017, 31:949-955.

16. Nutting C.M., Morden J.P., Harrington K.J. et al. Parotid-sparing intensity modulated versus conventional radiotherapy in head and neck cancer (PARSPORT): a phase 3 multicentre randomised controlled trial. Lancet Oncol 2011, 12:127-136.

17. Ghosh-Laskar S., Yathiraj P.H., Dutta D. et al. Prospective randomized controlled trial to compare 3-dimensional conformal radiotherapy to intensity-modulated radiotherapy in head and neck squamous cell carcinoma: Long-term results. Head Neck 2016, 38 Suppl 1:E1481-1487.

18. Peng G., Wang T., Yang K. et al. A prospective, randomized study comparing outcomes and toxicities of intensity-modulated radiotherapy vs. conventional two-dimensional radiotherapy for the treatment of nasopharyngeal carcinoma. Radiother Oncol 2012, 104:286-293.

19. Jirkovska M., Novak T., Malinova B. et al. Three-dimensional conformal radiotherapy versus intensity modulated radiotherapy with simultaneous integrated boost in the treatment of locally advanced head and neck carcinoma. Neoplasma 2019, 66:830-838.

20. Modesto A., Laprie A., Vieillevigne L. et al. Intensity-modulated radiotherapy for laryngeal and hypopharyngeal cancer: minimization of late dysphagia without jeopardizing tumor control. Strahlenther Onkol 2015, 191:225-233.

21. Mok G., Gauthier I., Jiang H. et al. Outcomes of intensity-modulated radiotherapy versus conventional radiotherapy for hypopharyngeal cancer. Head Neck 2015, 37:655-661.

22. Kılıç S., Kılıç S.S., Hsueh W.D. et al. Radiotherapy modality as a predictor of survival in hypopharyngeal cancer. Head Neck 2018, 40:2441-2448.

23. Weykamp F., Seidensaal K., Rieken S. et al. Age-dependent hemato- and nephrotoxicity in patients with head and neck cancer receiving chemoradiotherapy with weekly cisplatin. Strahlenther Onkol 2020, 196:515-521.

24. Ang K.K. Concurrent radiation chemotherapy for locally advanced head and neck carcinoma: are we addressing burning subjects? J Clin Oncol 2004, 22:4657-4659.

25. Szturz P., Wouters K., Kiyota N. et al. Weekly Low-Dose Versus Three-Weekly High-Dose Cisplatin for Concurrent Chemoradiation in Locoregionally Advanced Non-Nasopharyngeal Head and Neck Cancer: A Systematic Review and Meta-Analysis of Aggregate Data. Oncologist 2017, 22:1056-1066.

26. Guerrero Urbano T., Clark C.H., Hansen V.N. et al. A phase I study of dose-escalated chemoradiation with accelerated intensity modulated radiotherapy in locally advanced head and neck cancer. Radiother Oncol 2007, 85:36-41.

27. Schwartz M., Vuong T., Ballivy O. et al. Accelerated radiotherapy with simultaneous integrated boost fractionation and intensity-modulated radiotherapy for advanced head and neck cancer. Otolaryngol Head Neck Surg 2007, 136:549-555. 
28. Patel R.R., Ludmir E.B., Augustyn A. et al. De-intensification of therapy in human papillomavirus associated oropharyngeal cancer: A systematic review of prospective trials. Oral Oncol 2020, 103:104608.

29. Meade S., Gaunt P., Hartley A. et al. Feasibility of Dose-escalated Hypofractionated Chemoradiation in Human Papilloma Virus-negative or Smoking-associated Oropharyngeal Cancer. Clin Oncol (R Coll Radiol) 2018, 30:366-374.

30. Landis J., Koch G. The Measurement of Observer Agreement for Categorical Data. Biometrics 1977, 33:159-174.

31. Tao Y., Auperin A., Blanchard P. et al. Concurrent cisplatin and dose escalation with intensity-modulated radiotherapy (IMRT) versus conventional radiotherapy for locally advanced head and neck squamous cell carcinomas (HNSCC): GORTEC 2004-01 randomized phase III trial. Radiother Oncol 2020, 150:18-25.

32. Gupta T., Sinha S., Ghosh-Laskar S. et al. Intensity-modulated radiation therapy versus three-dimensional conformal radiotherapy in head and neck squamous cell carcinoma: long-term and mature outcomes of a prospective randomized trial. Radiat Oncol 2020, 15: 218. 